\title{
miR-143-3p inhibits endometriotic stromal cell proliferation and invasion by inactivating autophagy in endometriosis
}

\author{
HONG YANG, TIANQI HU, PANWEI HU, CONG QI and LIN QIAN \\ Department of Gynaecology, Shanghai Shuguang Hospital \\ Affiliated to Shanghai University of Traditional Chinese Medicine, Shanghai 201203, P.R. China
}

Received July 16, 2019; Accepted November 13, 2020

DOI: $10.3892 / \mathrm{mmr} .2021 .11995$

\begin{abstract}
Endometriosis (EM) is a multifactorial and debilitating chronic benign gynecological disease, but the pathogenesis of the disease is not completely understood. Dysregulated expression of microRNAs (miRNA/miR) is associated with the etiology of EM due to their role in regulating endometrial stromal cell proliferation and invasion. The present study aimed to identify the functions and mechanisms underlying miR-143-3p in EM. To explore the role of miR-143-3p in EM, functional miRNAs were analyzed via bioinformatics analysis. miR-143-3p expression levels in endometriotic stromal cells (ESCs) and normal endometrial stromal cells (NESCs) were measured via reverse transcription-quantitative PCR. The role of miR-143-3p in regulating ESC proliferation and invasion was assessed by performing Cell Counting Kit- 8 and Transwell assays, respectively. miR-143-3p expression was significantly upregulated in ESCs compared with NESCs. Functionally, miR-143-3p overexpression inhibited ESC proliferation and invasion, whereas miR-143-3p knockdown promoted ESC proliferation and invasion. Moreover, miR-143-3p inhibited autophagy activation in ESCs, as indicated by decreased green puncta, which represented autophagic vacuoles, decreased microtubule associated protein 1 light chain $3 \alpha$ expression and increased p62 expression in the miR-143-4p mimic group compared with the control group. Moreover, compared with the control group, miR-143-3p overexpression significantly decreased the expression levels of autophagy-related 2B (ATG2B), a newly identified target gene of miR-143-3p, in ESCs. ATG2B
\end{abstract}

Correspondence to: Dr Lin Qian, Department of Gynaecology, Shanghai Shuguang Hospital Affiliated to Shanghai University of Traditional Chinese Medicine, 528 Zhang Heng Road, Shanghai 201203, P.R. China

E-mail: qqqq19740@vip.sina.com

Abbreviations: miRNAs, microRNAs; EM, endometriosis; ESCs, endometriotic stromal cells; NESCs, normal endometrial stromal cells; ATG2B, autophagy-related 2B

Key words: endometriosis, endometriotic stromal cells, microRNA-143-3p, autophagy, ATG2B overexpression reversed miR-143-3p overexpression-mediated inhibition of ESC proliferation and invasion. Collectively, the results of the present study suggested that miR-143-3p inhibited EM progression, thus providing a novel target for the development of therapeutic agents against EM.

\section{Introduction}

Endometriosis (EM) is a common gynecological disease characterized by the growth of endometrial tissue outside the uterine cavity. EM affected $\sim 10 \%$ of women of reproductive age and 5-50\% of infertile women worldwide in 1997 (1). As a common gynecological disease, EM results in a number of clinical symptoms, including chronic pelvic pain, dysmenorrhea, dyspareunia, menorrhagia and mental suffering, which affect the quality of life of patients $(2,3)$. At present, the primary treatment strategy for EM involves relieving pain and other symptoms, but does not involve curing EM (4). Therefore, identifying the molecular mechanism underlying EM is important for the development of novel effective therapeutic strategies.

MicroRNAs (miRNA/miR) are non-coding short RNAs that modulate diverse life processes via regulating target genes at the post-transcriptional level (5). Previous studies identified abnormal expression of miRNAs in the eutopic endometrium, which indicated that miRNAs serve a key role in modulating the progression of EM (6-8). miR-143-3p is involved in cell proliferation, apoptosis, adhesion, invasion and other cellular processes $(9,10)$. Previous studies have reported that miR-143-3p was markedly dysregulated in EM (11-13).

Autophagy is a complex process, which is crucial for cellular self-regulation, and dysfunction of autophagy is associated with multiple human diseases, including cardiovascular diseases, neurodegeneration, metabolic diseases, infectious diseases and cancer (14-16). Several studies have demonstrated abnormal activation of autophagy in ovarian EM $(17,18)$. Therefore, the present study aimed to identify the functions and mechanisms underlying miR-143-3p in EM.

\section{Materials and methods}

Clinical samples, and cell isolation and culture. The present study was approved by the Protection of Human Subjects Committee of Shanghai Shuguang Hospital. All 
patients provided written informed consent. Ectopic $(\mathrm{n}=10)$, eutopic $(n=10)$ and normal endometrial $(n=10)$ tissues from patients with or without EM (mean age, 43.2 years; age range, 28-51 years) who had undergone a laparoscopy and uterine curettage were obtained from the Shanghai Shuguang Hospital Affiliated with Shanghai University of Traditional Chinese Medicine between November 2017 and March 2019. Endometriotic stromal cells (ESCs) were isolated from endometriotic tissues as previously described (19). Normal endometrial stromal cells (NESCs) were isolated from eutopic endometrial tissues obtained from four individuals without EM (age range, 30-53 years) who underwent a hysterectomy procedure. Briefly, the endometrium was minced and digested with collagenase type I (Gibco; Thermo Fisher Scientific, Inc.) for $45 \mathrm{~min}$ at room temperature. After filtrating through a stainless wire mesh $(200 \mu \mathrm{m})$ and gentle centrifugation at $250 \mathrm{x} \mathrm{g}$ for $25 \mathrm{~min}$ at room temperature, ESCs or NESCs were isolated via passing over a stainless wire mesh $(400 \mu \mathrm{m})$. Subsequently, resuspended cells were layered over Ficoll (Beijing Solarbio Science \& Technology Co., Ltd.), centrifuged at 2,000 x g for $25 \mathrm{~min}$ at room temperature and cells in the middle layer were collected. ESCs or NESCs were cultured in DMEM/F-12 (Gibco; Thermo Fisher Scientific, Inc.) supplemented with 10\% FBS (HyClone; GE Healthcare Life Sciences) with $5 \% \mathrm{CO}_{2}$ at $37^{\circ} \mathrm{C}$.

EM mouse model, and isolation of ESCs and NESCs. The EM mouse model was established using C57BL/6 female mice (age, 8 weeks; weight, 25-30 g; $n=10 ; n=5$ per group; Shanghai Model Organisms Center, Inc.). Mice were divided into two groups ( $=5$ per group): i) EM and ii) Control. All experimental procedures were approved by the Ethics Committee for Animal Experimentation of Shanghai Shuguang Hospital. Mice were housed in a barrier unit in a sterile environment at $22^{\circ} \mathrm{C}$ with 40-80\% humidity, 12-h light/dark cycles, and ad libitum access to food and water. Mice were anesthetized with an intraperitoneal injection of chloral hydrate $(350 \mathrm{mg} / \mathrm{kg})$. Animals did not exhibit signs of peritonitis following the administration of chloral hydrate. Endometrial pieces $\left(1 \mathrm{~mm}^{3}\right)$ isolated from ovarian endometriotic samples from patients with EM were suspended in saline and $400 \mu 1$ suspension was injected into the peritoneal cavity of the mice. At 3 weeks after model establishment, mice were euthanized by cervical dislocation. Sections of endometrial tissue were isolated from control mice and EM model mice. Tissue sections were washed twice with PBS and cultured in DMEM/F12 medium supplemented with 20\% FBS at $37^{\circ} \mathrm{C}$ with $5 \% \mathrm{CO}_{2}$. The culture medium was changed with the appearance of a large number of desquamated endothelial cells (every 3 days). Non-adherent cells were removed carefully. At $80-90 \%$ confluence, the culture medium was removed, cells were washed twice with PBS and then treated with $0.25 \%$ trypsin. Subsequently, the cell concentration was adjusted to $1.0 \times 10^{6} / \mathrm{ml}$ using DMEM medium supplemented with $10 \%$ FBS. Cells were cultured in DMEM/F12 supplemented with $10 \% \mathrm{FBS}$ at $37^{\circ} \mathrm{C}$ with $5 \% \mathrm{CO}_{2}$.

Transfection. miR-143-3p mimic (5'-UGAGAUGAAGCACUG UAGCUC-3'), 2'-O-methyl-modified anti-miR-143-3p (5'-GAG CUACAGUGCUUCAUCUCA-3'), miR-143-3p mimic negative control (NC; 5'-UCACAACCUCCUAGAAAGAGUAGA-3') and anti-miR-143-3p NC (5'-UACUCUUUCUAGGAGGUU GUGAUU-3') were obtained from Shanghai GenePharma Co., Ltd. Cells $\left(1 \times 10^{6}\right)$ were transfected with $20 \mathrm{ng} / \mathrm{ml} \mathrm{miR-143-3p}$ mimic, anti-miR-143-3p, miR-143-3p mimic NC or anti-miR-143-3p NC using Lipofectamine ${ }^{\circledR} 2000$ (Invitrogen; Thermo Fisher Scientific, Inc.) for $48 \mathrm{~h}$ at $37^{\circ} \mathrm{C}$. At $48 \mathrm{~h}$ post-transfection, cells were collected for subsequent experiments.

Total RNA was extracted from 293T cells using TRIzol ${ }^{\circledR}$ reagent (Takara Bio, Inc.). Total RNA was reverse transcribed into cDNA using the PrimeScript ${ }^{\mathrm{TM}} \mathrm{RT}$ reagent kit (Takara Bio, Inc.). The full-length cDNA of Autophagy-related 2B (ATG2B) was cloned into the pcDNA3.1 vector (Invitrogen; Thermo Fisher Scientific, Inc.). The sequences of the primers used to amplify ATG2B were as follows: forward, 5'-GGAGCCACTCTCCAGCATAG-3' and reverse, 5'-GTG CACAGCTCCAAAGATGA-3'. The following thermocycling conditions were used: Incubation at $50^{\circ} \mathrm{C}$ for $2 \mathrm{~min} ; 95^{\circ} \mathrm{C}$ for $2 \mathrm{~min}$; followed by 40 cycles of $95^{\circ} \mathrm{C}$ for $15 \mathrm{sec}$ and $60^{\circ} \mathrm{C}$ for $32 \mathrm{sec}$. NESCs were seeded into multiple-well plates at $\sim 80 \%$ confluence. Cells were transfected with recombinant plasmids (1.5 $\mu \mathrm{g}$ per well) using Lipofectamine ${ }^{\circledR} 2000$ (Invitrogen) at room temperature for $6 \mathrm{~h}$ according to the manufacturer's protocol. At $48 \mathrm{~h}$ post-transfection, subsequent experiments were performed. pcDNA3.1 was used as a negative control.

Luciferase reporter assay. To confirm the potential target genes of miR-143-3p in ESCs, the present study searched for candidate genes using TargetScan (version 7.1; www. targetscan.org/vert_71) and miRBase22 (www.mirbase. org) databases. A 3'-untranslated region (UTR) luciferase reporter vector of ATG2B containing the predicted binding sites of miR-143-3p was produced by cloning the 3'-UTR of the corresponding mRNA into the pGL3-promoter vector (Promega Corporation). Subsequently, 293T cells $\left(1 \times 10^{4}\right)$ were co-transfected with $1 \mathrm{ng}$ pRL-TK (Promega Corporation), $100 \mathrm{ng}$ pGL3-ATG2B-3'-UTR-wild-type (WT) or pGL3-ATG2B-3'-UTR-mutant (mut) and $20 \mathrm{nM}$ miR-143-3p mimic or miR-143-3p mimic NC using Lipofectamine ${ }^{\circledR} 3000$ (Thermo Fisher Scientific, Inc.). At 72 h post-transfection, luciferase activities were measured using a Dual-Luciferase Reporter Assay System (Promega Corporation) according to the manufacturer's protocol. Firefly luciferase activity was normalized to Renilla luciferase activity.

Immunofluorescence. Autophagy vacuoles were assessed using an Autophagy Detection kit (cat. no. ab139484; Abcam) according to the manufacturer's protocol. A coverslip was placed into each well of a 24-well plate. ESCs were seeded $\left(2 \times 10^{4}\right)$ into the 24 -well plate and incubated at $37^{\circ} \mathrm{C}$ for 1 day. Following washing three times with PBS, cells were fixed with $4 \%$ cold paraformaldehyde for $15 \mathrm{~min}$ and then washed three times with PBS. After blocking with $1 \%$ BSA (Sigma-Aldrich; Merck KGaA) for $30 \mathrm{~min}$ at room temperature, the fluorescent dyes for nuclei staining and autophagy detection were added and incubated for $30 \mathrm{~min}$ at room temperature. After washing three times using PBS, the slides were observed using a FV1000s-SIM/IX81 confocal laser scanning microscope (Olympus Corporation). The ratio of green to blue fluorescence was calculated to assess the degree of autophagy. 

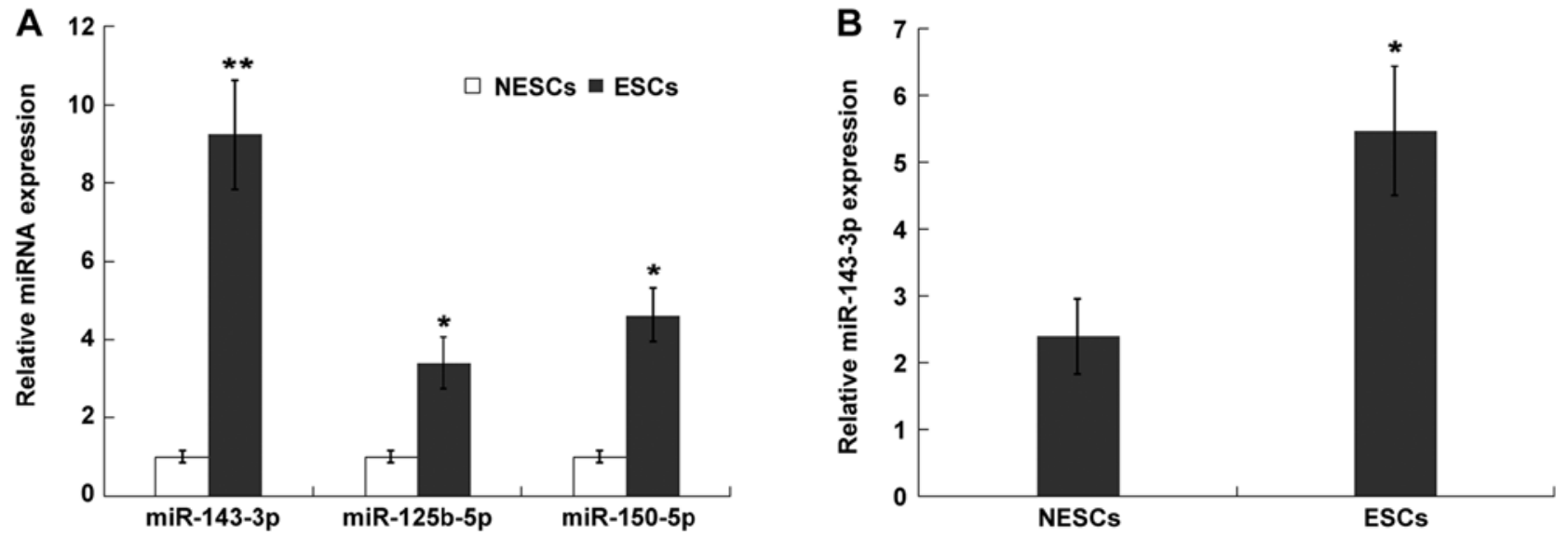

Figure 1. miR-143-3p is significantly upregulated in ESCs. (A) miR-143-3p, miR-125b-5p and miR-449b-5p expression levels in ESCs and NESCs. (B) miR-143-3p expression levels in ESCs derived from endometriosis model mice and NESCs derived from healthy control mice. ${ }^{*} \mathrm{P}<0.05$ and ${ }^{* *} \mathrm{P}<0.01$ vs. NESCs. miR, microRNA; ESC, endometriotic stromal cell; NESC, normal endometrial stromal cell.

$R N A$ isolation and reverse transcription quantitative PCR $(q P C R)$. Total RNA was extracted from ESCs and NESCs using TRIzol ${ }^{\circledR}$ reagent (Takara Bio, Inc.). Total RNA was reverse transcribed into cDNA using the PrimeScript ${ }^{\mathrm{TM}}$ RT reagent kit (Takara Bio, Inc.) at $37^{\circ} \mathrm{C}$. Subsequently, qPCR was performed using SYBR-Green PCR Master Mix (Takara Bio, Inc.) on an ABIStep One Plus ${ }^{\mathrm{TM}}$ real-time PCR System (Applied Biosystems; Thermo Fisher Scientific, Inc.). The following thermocycling conditions were used for qPCR: $95^{\circ} \mathrm{C}$ for $10 \mathrm{~min}$; followed by 35 cycles of $95^{\circ} \mathrm{C}$ for $10 \mathrm{sec}, 58^{\circ} \mathrm{C}$ for $15 \mathrm{sec}$ and $72^{\circ} \mathrm{C}$ for $20 \mathrm{sec}$; and final extension at $72^{\circ} \mathrm{C}$ for $20 \mathrm{~min}$. The following primers were used for qPCR: miR-143-3p forward, 5'-CTGGCGTTGA GATGAAGCAC-3' and reverse, 5'-CAGAGCAGGGTCCGAG GTA-3'; miR-125b-5p forward, 5'-TCCCTGAGACCCTAACT TGTGA-3' and reverse, 5'-AGTCTCAGGGTCCGAGGTA TTC-3'; miR-150-5p forward, 5'-TCGGCGTCTCCCAACCC TTGTAC-3' and reverse, 5'-GTCGTATCCAGTGCAGGGTC CGAGGT-3'; ATG2B forward, 5'-TCCTTCAGGAAGAACAAA GCA-3' and reverse, 5'-AAGCCTTACACGTGTGTCCA-3'; U6 forward, 5'-CTCGCTTCGGCAGCACA-3' and reverse, 5'-AAC GCTTCACGAATTTGCGT-3'; and GAPDH forward, 5'-ATT CCACCCATGGCAAATTC-3' and reverse, 5'-TGGGATTTC CATTGATGACAAG-3'. miRNA and mRNA expression levels were quantified using the $2^{-\Delta \Delta \mathrm{Cq}}$ method (20) and normalized to the internal reference genes U6 and GAPDH, respectively.

Western blotting. Total protein was extracted from ESCs using RIPA buffer (Cell Signaling Technology, Inc.). Total protein was quantified using a BCA kit (Takara Bio, Inc.). Proteins $(20 \mu \mathrm{g})$ were separated via 12\% SDS-PAGE and transferred to PVDF membranes. After blocking with 5\% BSA (in TBS $/ 0.05 \%$ Tween-20 buffer) for $2 \mathrm{~h}$ at room temperature, the membranes were incubated at $4^{\circ} \mathrm{C}$ overnight with primary antibodies (all purchased from Abcam) targeted against the following: ATG2B (1:5,000; cat. no. ab226832), p62 (1:1,000; cat. no. ab109012) and microtubule associated protein 1 light chain $3 \alpha$ (LC3)-I/II (1:2,000; cat. no. ab128025) and actin (1:5,000; cat. no. ab8226). Following primary incubation, the membranes were incubated with a Goat Anti-Mouse IgG H\&L (HRP) secondary antibody (1:2,000; cat. no. ab205719; Abcam) for $1 \mathrm{~h}$ at room temperature. Protein bands were visualized using an ECL kit (Pierce; Thermo Fisher Scientific, Inc.). Protein expression levels were semi-quantified using the Odyssey Infrared Imaging System (version 3.0; LI-COR Biosciences) with actin as the loading control.

Transwell assays. A Transwell assay was performed to assess cell invasion. The Transwell insert (pore size, $8 \mu \mathrm{m}$; Corning Life Sciences) was precoated with Matrigel for $4 \mathrm{~h}$ at $37^{\circ} \mathrm{C}$. ESCs were seeded $\left(5 \times 10^{4}\right)$ into the upper chamber in serum-free medium and DMEM/F-12 medium supplemented with $10 \%$ FBS was plated into the lower chamber. Cells were incubated at $37^{\circ} \mathrm{C}$ with $5 \% \mathrm{CO}_{2}$ for 1 day. Subsequently, invading cells were fixed with $4 \%$ paraformaldehyde for 40 min at room temperature and stained with $1 \%$ crystal violet staining solution for $15 \mathrm{~min}$ at room temperature. Stained cells were quantified by counting the number of cells in five randomly selected fields of view using a light microscope.

Cell proliferation assay. Cell proliferation was assessed by conducting a Cell Counting Kit-8 (CCK-8) assay (Dojindo Molecular Technologies, Inc.) according to the manufacturer's protocol. Briefly, cells were seeded $\left(5 \times 10^{3}\right.$ cells/well $)$ into 96-well plates. Subsequently, at 0, 24, 48 and 72 h, $10 \mu \mathrm{l} \mathrm{CCK-8}$ reagent was added to each well and incubated for $4 \mathrm{~h}$. The absorbance was measured at a wavelength of $450 \mathrm{~nm}$ using a VersaMax microplate reader (Molecular Devices, LLC).

Statistical analysis. Data are presented as the mean \pm SD. All experiments were repeated at least three times. Statistical analyses were performed using SPSS software (version 15; SPSS, Inc.). Comparisons between two groups were analyzed using the Mann-Whitney U test (Fig. 1) or unpaired Student's t-test (Figs. 2, 3, and 4B, C and F). Comparisons among multiple groups were analyzed using one-way ANOVA followed by Scheffé's post hoc test (Fig. 4D and E). P<0.05 was considered to indicate a statistically significant difference.

\section{Results}

miR-143-3p is markedly upregulated in ESCs. A number of studies have demonstrated that miRNAs participate in the 
A
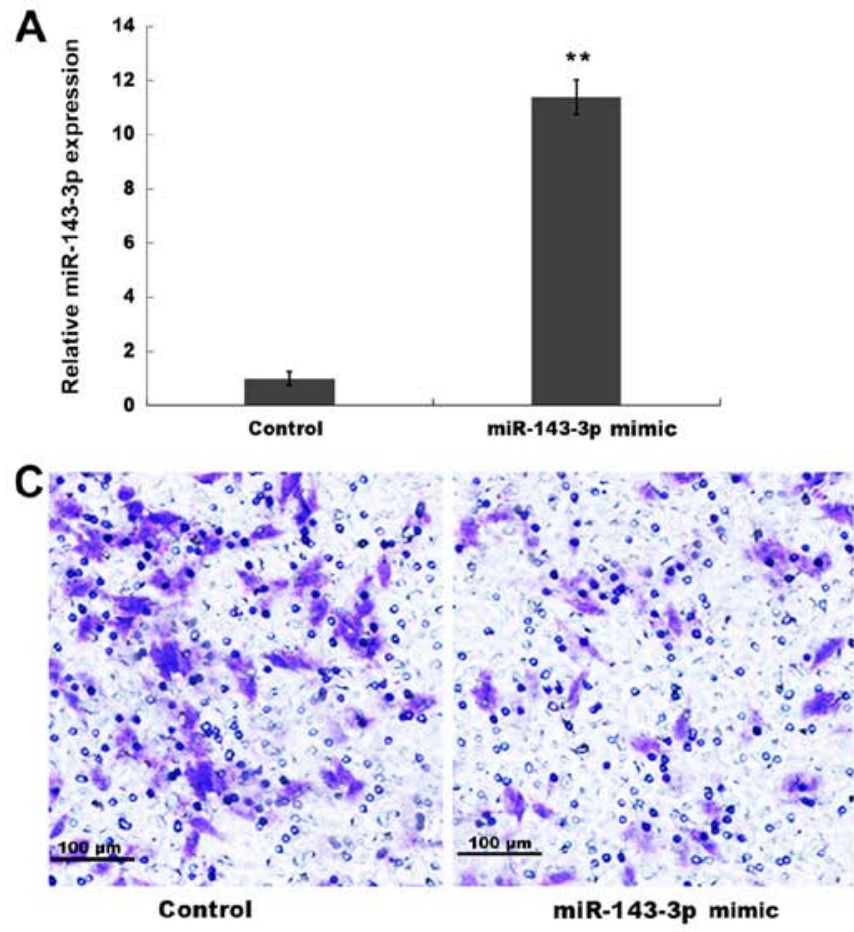

\section{E}

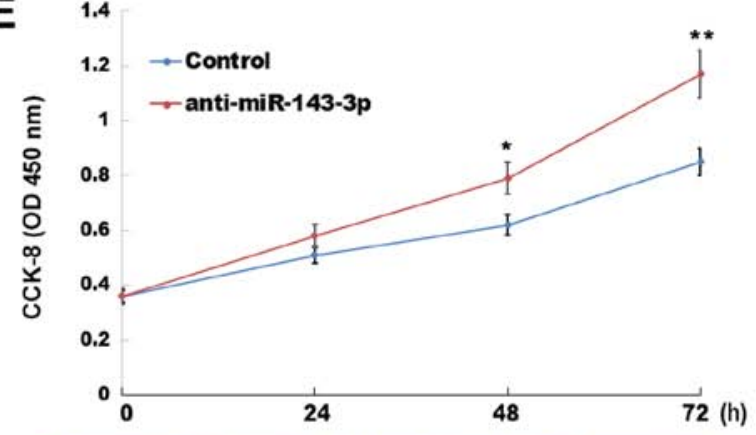

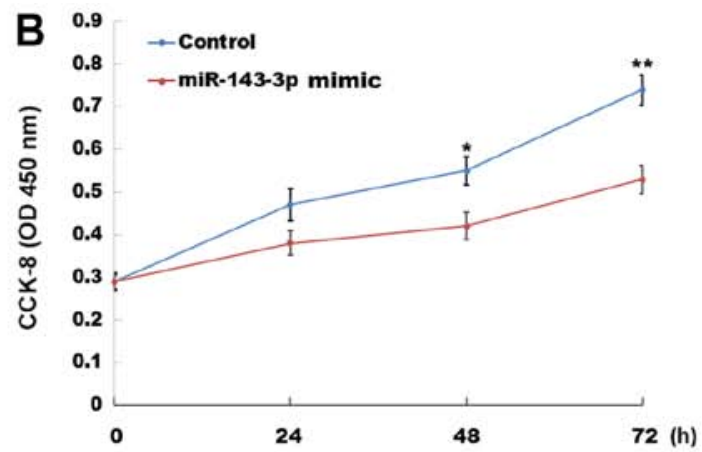
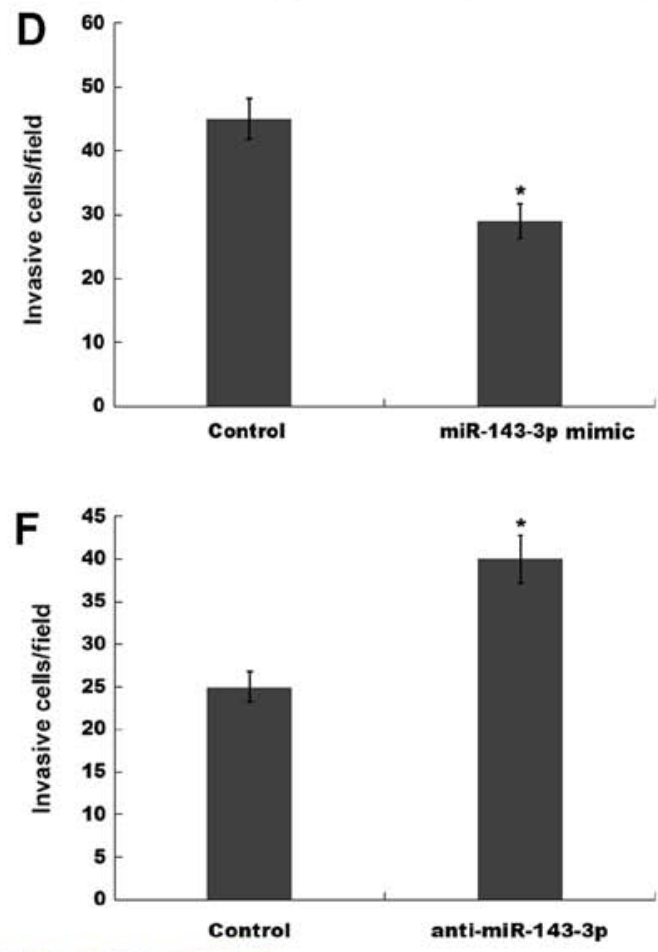

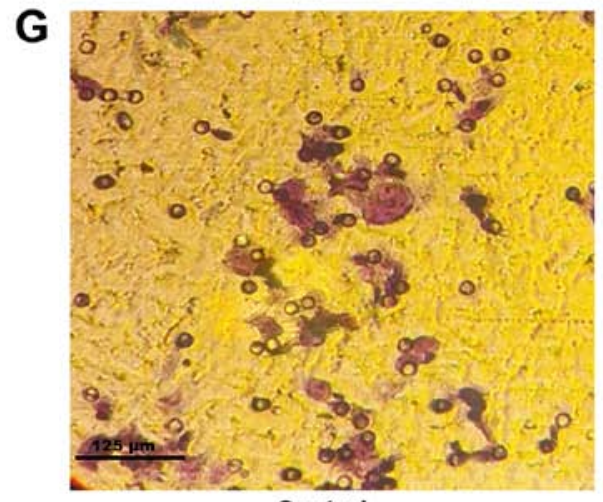

Control

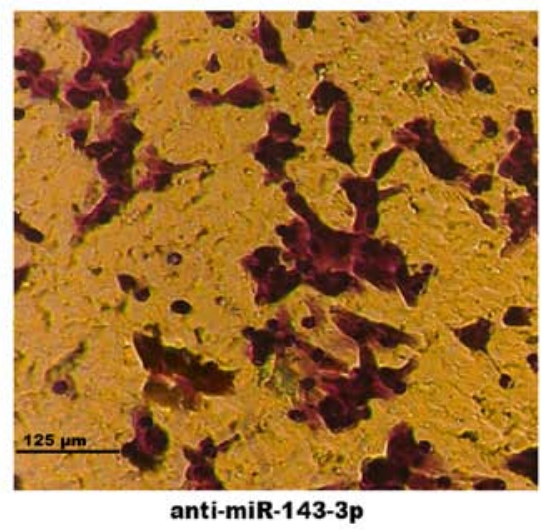

Figure 2. miR-143-3p inhibits ESC proliferation and invasion. (A) Transfection efficiency of miR-143-3p mimic. (B) Cell proliferation in miR-143-3p mimic-transfected ESCs was assessed by performing the CCK-8 assay. Cell invasion was (C) assessed by performing a Transwell invasion assay and (D) quantified. (E) Cell proliferation in anti-miR-143-3p-transfected ESCs was assessed by performing the CCK-8 assay. (F) Cell invasion was assessed by performing a Transwell invasion assay. (G) Representative images of the Transwell invasion assay. ${ }^{*} \mathrm{P}<0.05$ and ${ }^{* *} \mathrm{P}<0.01$ vs. control. miR, microRNA; ESC, endometriotic stromal cell; CCK-8, Cell Counting Kit-8; OD, optical density.

development and progression of EM via multiple mechanisms (21,22). Abdel-Rasheed et al (23) reported that 32 miRNAs were significantly dysregulated in EM and Cosar et al (13) demonstrated that there are 10 miRNAs that may serve as diagnostic markers of EM. The present study re-analyzed the expression level of three upregulated miRNAs (miR-125b-5p, miR-150-5p and miR-143-3p) that appeared in both datasets (Fig. S1A). The result was verified in ESCs and NESCs. The expression levels of miR-125b-5p, miR 150-5p and miR-143-3p were significantly increased in ESCs compared with NESCs (Fig. 1A). In the present study, as miR-143-3p was the most upregulated miRNA among the three miRNAs in ESCs, miR-143-3p was further investigated. Previous studies have also demonstrated that miR-143-3p is 

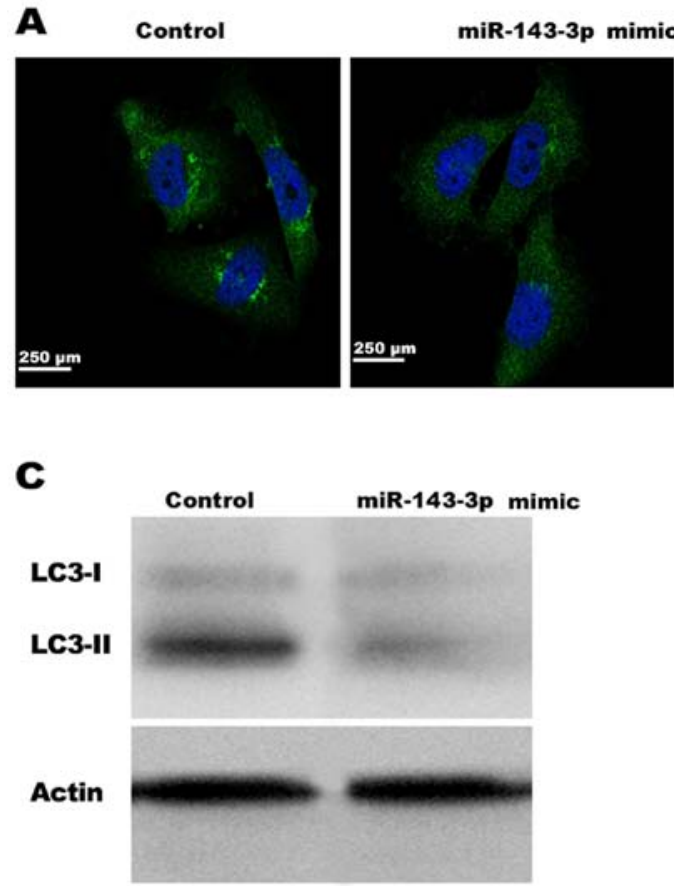

$\mathbf{E}$

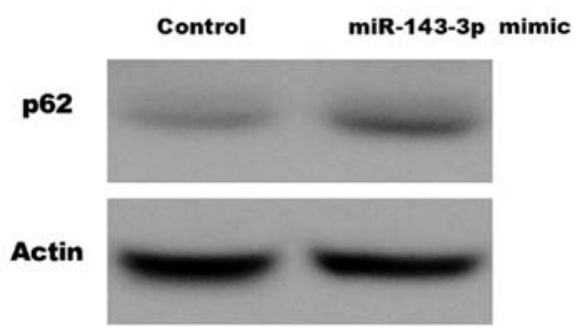

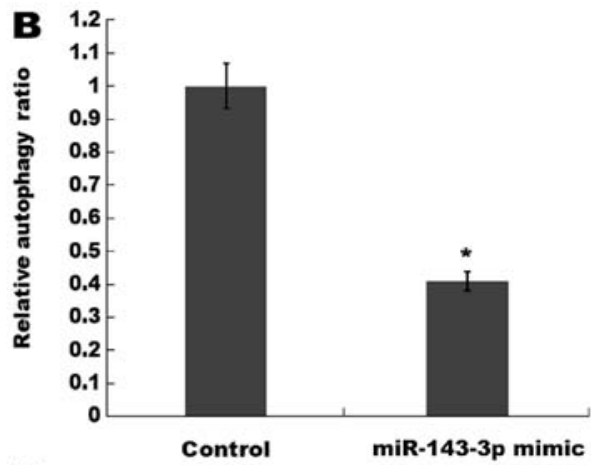
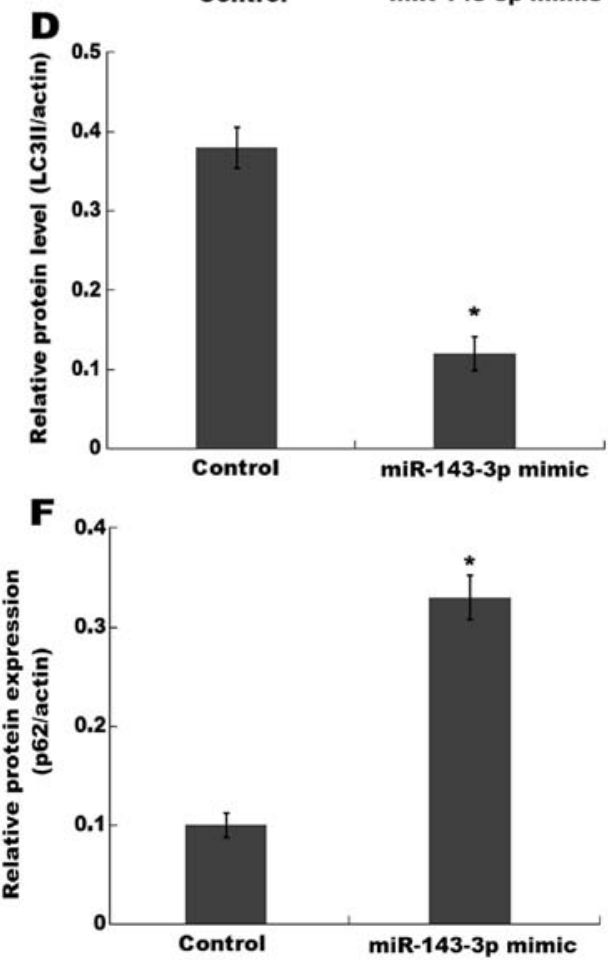

Figure 3. miR-143-3p suppresses autophagy activation in endometriotic stromal cells. Autophagic vacuoles were identified via (A) immunofluorescence staining and (B) the autophagy ratio was calculated (ratio of green fluorescence to blue fluorescence). LC3-II protein expression levels were determined via (C) western blotting and (D) semi-quantified. p62 protein expression levels were determined via (E) western blotting and (F) semi-quantified. ${ }^{*} \mathrm{P}<0.05$ vs. control. miR, microRNA; LC3, microtubule associated protein 1 light chain $3 \alpha$.

upregulated in ectopic endometrial tissues compared with eutopic endometrial tissues $(7,24)$. To further verify the in vitro results, the present study established an EM mouse model and assessed miR-143-3p expression levels in isolated ESCs. miR-143-3p expression levels were significantly increased in ESCs compared with NESCs (Fig. 1B).

miR-143-3p inhibits ESC proliferation and invasion. The role of miR-143-3p in ESC proliferation and invasion was investigated in the present study. miR-143-3p was overexpressed in ESCs and subsequently, cell proliferation and invasion were assessed by performing CCK- 8 and Transwell invasion assays, respectively. miR-143-3p mimic significantly increased miR-143-3p expression levels in ESCs compared with the control group (Fig. 2A). The CCK-8 assay results indicated that miR-143-3p overexpression also significantly suppressed ESC proliferation at the 48 and $72 \mathrm{~h}$ time points compared with the control group (Fig. 2B). The present study also assessed the role of miR-143-3p overexpression in ESC invasion. The Transwell invasion assay results indicated that miR-143-3p overexpression significantly inhibited ESC invasion compared with the control group (Fig. 2C and D). By contrast, compared with the control group, miR-143-3p knockdown significantly enhanced ESC proliferation at 48 and $72 \mathrm{~h}$, and significantly increased ESC invasion (Figs. S1B and 2E-G). The aforementioned results suggested that miR-143-3p overexpression inhibited EM progression.

miR-143-3p suppresses autophagy activation in ESCs. Previous studies have observed autophagy activation in ectopic endometrium of patients with ovarian endometriosis $(17,25,26)$. Based on the finding that miR-143-3p displays the potential to regulate autophagy in other diseases $(27,28)$, the present study investigated whether miR-143-3p inhibited ESC proliferation and invasion via inactivating autophagy. The activation of autophagy was analyzed using an autophagy detection kit and western blotting. Compared with the control group, miR-143-3p overexpression significantly decreased the autophagy ratio, as evidenced by a decreased number of green puncta, which represented autophagic vacuoles (Fig. 3A and B). miR-143-3p 
A

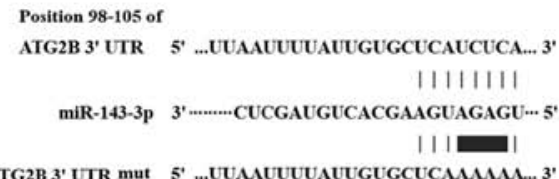

B
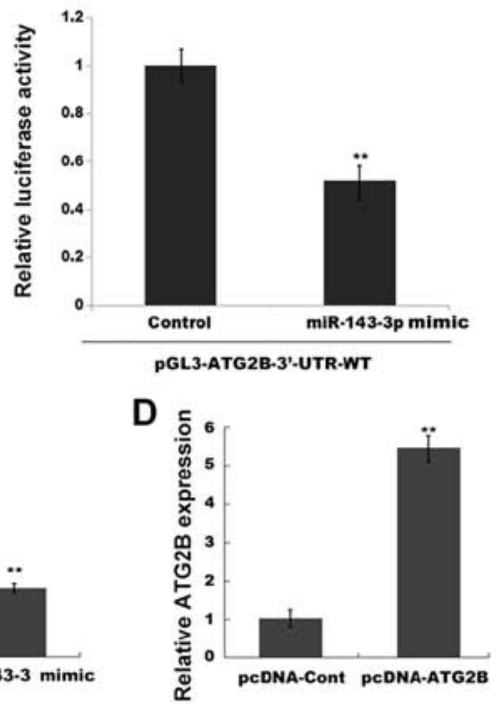

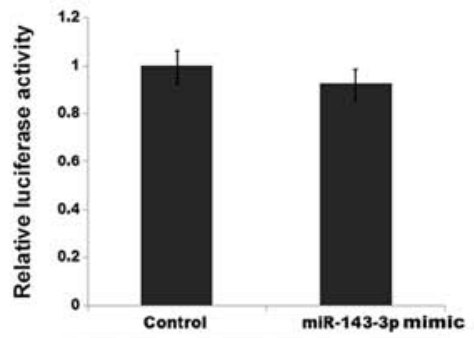

PGL3-ATG2B-3'-UTR-mut

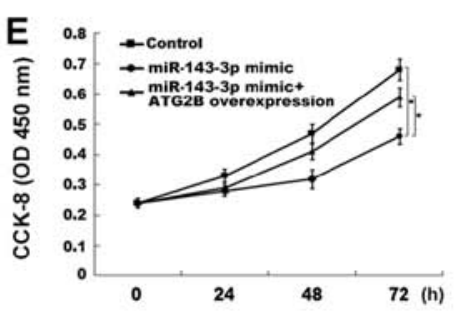

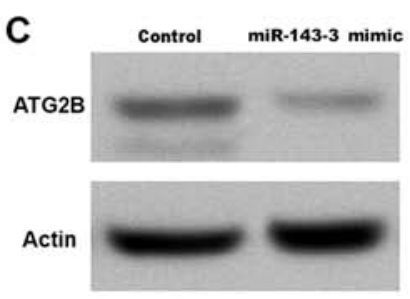
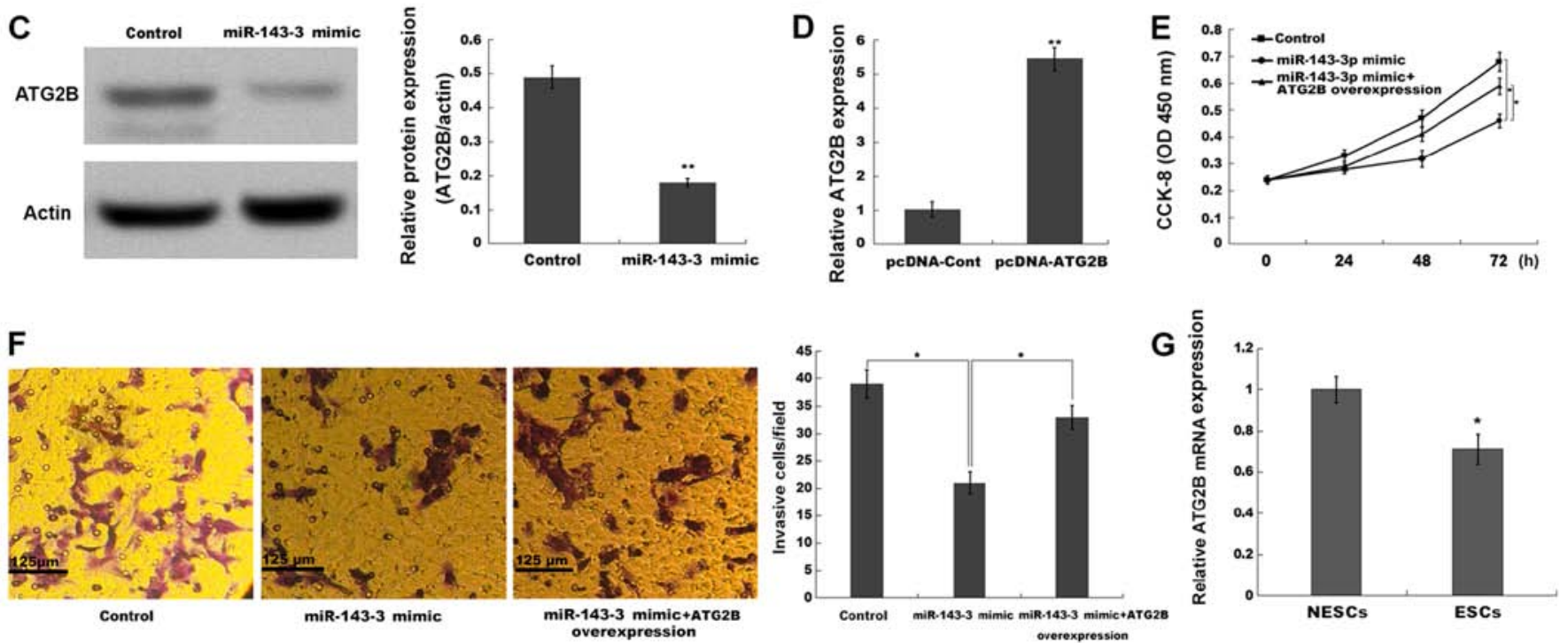

Figure 4. miR-143-3p inhibits ESC proliferation and invasion by repressing ATG2B. (A) The binding sites between miR-143-3p and the 3'-UTR of ATG2B. (B) The luciferase reporter assay was performed in ESCs co-transfected with miR-143-3p mimic or negative control, pGL3-ATG2B-3'-UTR-WT or pGL3-ATG2B-3'-UTR-mut and pRL-TK. ${ }^{* *} \mathrm{P}<0.001$ vs. control. (C) ATG2B protein expression levels were determined via western blotting and semi-quantified. (D) Transfection efficiency of pcDNA-ATG2B. Compared with the control group, miR-143-3p overexpression repressed ESC (E) proliferation and (F) invasion, whereas ATG2B overexpression reversed miR-143-3p-mimic mediated effects. (G) ATG2B mRNA expression levels in ESCs and NESCs isolated from endometriosis model mice. ${ }^{*} \mathrm{P}<0.05$ and ${ }^{* *} \mathrm{P}<0.01$ vs. control or NESCs. miR, microRNA; ESC, endometriotic stromal cell; ATG2B, autophagy-related $2 \mathrm{~B}$; UTR, untranslated region; WT, wild-type; mut, mutant; CCK-8, Cell Counting Kit-8; OD, optical density; NESC, normal endometrial stromal cell; cont, control.

overexpression also significantly decreased LC3-II expression levels (a reliable indicator of autophagy) in ESCs compared with the control group, indicating inhibition of autophagy (Fig. 3C and D). Furthermore, miR-143-3p overexpression significantly increased p62 (an autophagy substrate) protein expression levels in ESCs compared with the control group (Fig. 3E and F). The results indicated that miR-143-3p overexpression inhibited autophagy activation in ESCs.

miR-143-3p suppresses ESC proliferation and invasion by repressing $A T G 2 B$. To confirm the potential target genes of miR-143-3p in ESCs, the present study searched for candidate genesusing TargetScan(version 7.1; www.targetscan.org/vert_71) and miRBase22 (www.mirbase.org) databases. Bioinformatics analysis using TargetScan and miRBase identified 487 potential target genes. Among the identified target genes, ATG2B was the only gene associated with the autophagy signaling pathway (29). The bioinformatics analysis indicated that miR-143-3p directly targeted the 3'-UTR of the ATG2B gene, an essential regulator of autophagy activation (30) (Fig. 4A). To verify whether miR-143-3p directly bound to the 3'-UTR of ATG2B and repressed its expression, the present study constructed a luciferase reporter vector containing the 3'-UTR of ATG2B. The results indicated that miR-143-3p mimic significantly inhibited the luciferase activity of pGL3-ATG2B-3'-UTR-WT compared with the control group, whereas miR-143-4p mimic displayed no significant effect on pGL3-ATG2B-3'-UTR-mut compared with the control group (Fig. 4B). Furthermore, miR-143-3p overexpression significantly decreased the protein expression levels of ATG2B in ESCs compared with the control group (Fig. 4C). In addition, compared with the control group, miR-143-3p overexpression significantly inhibited ESC proliferation and invasion, whereas ATG2B overexpression significantly alleviated miR-143-3p mimic-mediated effects (Fig. 4D-F). The present study also assessed the expression levels of ATG2B in ESCs derived from the EM mouse model. The results indicated that ATG2B expression levels were significantly decreased in ESCs compared with NESCs (Fig. 4G). Collectively, the aforementioned results suggested that miR-143-3p overexpression inhibited EM progression by repressing ATG2B expression, thus inactivating autophagy.

\section{Discussion}

In the present study, the effect of miRNAs on regulating EM progression was investigated. miR-143-3p expression was significantly upregulated in ESCs compared with NESCs. miR-143-3p overexpression markedly decreased ESC cell proliferation and invasion compared with the control group. In addition, compared with the control group, miR-143-3p 
overexpression significantly decreased LC3-II expression levels and increased p62 expression levels, indicating that miR-143-3p may serve as an inhibitor of autophagy activation in ESCs. Furthermore, the present study verified that miR-143-3p directly targeted the 3'-UTR of ATG2B, and miR-143-3p overexpression significantly decreased the protein expression levels of ATG2B compared with the control group. ATG2B overexpression partially reversed miR-143-3p mimic-mediated effects on ESC proliferation and invasion.

As a gynecological disease, EM frequently results in infertility and chronic pelvic pain (31). Immune disorders affect ectopic endometrial lesions. Following dysfunction of the immune system, the number of immune cells increases, and various growth factors, cytokines, non-specific immunoglobulins and proinflammatory mediators are present in the peritoneum (32-34). A theory of EM is the local hypoxia microenvironment, whereby the first indicator of EM is the topical shifted hypoxic microenvironment, when the shed endometrial fragments retrograde to the peritoneal cavity (35-37).

Autophagy is a highly conserved cellular process, which is activated by various factors, such as hypoxia (38). For the past few years, research has focused on autophagy progression in tumorigenesis $(39,40)$. EM displays certain biological characteristics of tumor diseases, such as hyperproliferation and metastasis (41) Increasing evidence has demonstrated that autophagy is dysregulated in the uterine horns and eutopic endometria of EM model mice (42), and is correlated with endometrial regulation and the pathophysiology of EM $(43,44)$. However, the role of autophagy in EM is controversial, thus whether autophagy in EM is beneficial or detrimental remains to be elucidated. Several studies have demonstrated that the expression levels of autophagy-related genes (for example, Beclin-1 and LC3-II) are markedly decreased and autophagy activation is downregulated in endometrial stromal cells of patients with EM compared with healthy controls (45-47). Functionally, autophagy inhibition contributes to endometrial cell invasion, whereas autophagy activation represses ESC proliferation, colony formation and invasion (48). By contrast, other studies have reported that the expression levels of autophagy-associated genes [for example, Beclin-1, autophagy-related (ATG)14, ATG7 and LC3-II] are increased and autophagy activation is upregulated in ESCs in EM (17). Liu et al (44) further demonstrated that HIF-1 $\alpha$ facilitates endometrial stromal cell invasion by activating autophagy, whereas autophagy inhibition alleviates hypoxia-induced cell invasion. The present study demonstrated that miR-143-3p overexpression inhibited ESC proliferation and invasion by regulating ATG2B in EM, whereas ATG2B overexpression partially reversed miR-143-3p overexpression-mediated effects, indicating that autophagy may be beneficial in EM. Although the effect of miR-143-3p on inhibiting autophagy has been verified in Crohn's disease (27), the present study aimed to investigate the association of miR-143-3p with ATG2B and autophagy in EM. The present study further clarified the function of miR-143-3p in EM. However, a key limitation of the present study was that the role of miR-143-3p in NESC autophagy was not investigated.

A previous study demonstrated that miRNAs are crucial modulators in the occurrence and development of various diseases (49-52). Several studies have implicated that the aberrant expression of miRNAs is a potential regulator of EM pathogenesis $(11,23)$. It has been verified that the expression levels of HIF-1 $\alpha$ and VEGF were elevated in ectopic endometrial tissues compared with eutopic endometrial tissues, which was induced by hypoxia stress. $\mathrm{miR}-17-5 \mathrm{p} / 20 \mathrm{a}$ is a regulator of HIF-1 $\alpha$ and VEGF via directly targeting the 3'-UTR, and further regulates downstream hypoxic stress-associated proteins (53-55). The present study investigated the function of miR-143-3p in EM and demonstrated that miR-143-3p overexpression notably inhibited ESC proliferation and invasion in EM compared with the control group. In hepatocellular carcinoma, upregulated miR-143-3p promotes cancer cell migration and invasion by repressing fibronectin type III domain containing 3B (56). Therefore, the results of the present study and the aforementioned previous studies suggested that miR-143-3p may serve various regulatory roles in different biological processes or diseases.

In summary, miR-143-3p was significantly upregulated in ESCs compared with NESCs. miR-143-3p regulated the phenotype of EM, suppressing autophagy activation in ESCs, and inhibiting ESC proliferation and invasion by directly targeting ATG2B. The results of the present study may further the current understanding of the role of miR-143-3p in the pathogenesis of EM.

\section{Acknowledgements}

Not applicable.

\section{Funding}

The present study was supported by the National Natural Science Foundation of China (grant no. 81704108).

\section{Availability of data and materials}

The datasets used and/or analyzed during the current study are available from the corresponding author on reasonable request.

\section{Authors' contributions}

HY and LQ made substantial contributions to the conception and design of the present study. TH, PH, CQ and LQ collected, analyzed and interpreted the data. PH, CQ and LQ drafted the work and made critical modifications to the manuscript. All authors agreed to be accountable for the work in ensuring that questions related to the integrity of any part of the work are appropriately investigated and resolved. All authors read and approved the final manuscript.

\section{Ethics approval and consent to participate}

The present study was approved by the Protection of Human Subjects Committee of Shanghai Shuguang Hospital and the Ethics Committee for Animal Experimentation of Shanghai Shuguang Hospital (approval no. 2018-618-47-01). All patients or their legal guardians provided written informed consent.

\section{Patient consent for publication}

Not applicable. 


\section{Competing interests}

The authors declare that they have no competing interests.

\section{References}

1. Eskenazi B and Warner ML: Epidemiology of endometriosis Obstet Gynecol Clin North Am 24: 235-258, 1997.

2. Simoens S, Dunselman G, Dirksen C, Hummelshoj L, Bokor A, Brandes I, Brodszky V, Canis M, Colombo GL, DeLeire T, et al: The burden of endometriosis: Costs and quality of life of women with endometriosis and treated in referral centres. Hum Reprod 27: 1292-1299, 2012.

3. Shafrir AL, Farland LV, Shah DK, Harris HR, Kvaskoff M, Zondervan K and Missmer SA: Risk for and consequences of endometriosis: A critical epidemiologic review. Best Pract Res Clin Obstet Gynaecol 51: 1-15, 2018.

4. Friend DR: Drug delivery for the treatment of endometriosis and uterine fibroids. Drug Deliv Transl Res 7: 829-839, 2017.

5. Vishnoi A and Rani S: miRNA biogenesis and regulation of diseases: An overview. Methods Mol Biol 1509: 1-10, 2017.

6. Arora S, Rana R, Chhabra A, Jaiswal A and Rani V: miRNA-transcription factor interactions: A combinatorial regulation of gene expression. Mol Genet Genomics 288: 77-87, 2013.

7. Filigheddu N, Gregnanin I, Porporato PE, Surico D, Perego B Galli L, Patrignani C, Graziani A and Surico N: Differential expression of microRNAs between eutopic and ectopic endometrium in ovarian endometriosis. J Biomed Biotechnol 2010: 369549, 2010.

8. Zhao L, Gu C, Ye M, Zhang Z, Li L, Fan W and Meng Y: Integration analysis of microRNA and mRNA paired expression profiling identifies deregulated microRNA-transcription factor-gene regulatory networks in ovarian endometriosis. Reprod Biol Endocrinol 16: 4, 2018.

9. Shi H, Shen H, Xu J, Zhao S, Yao S and Jiang N: MiR-143-3p suppresses the progression of ovarian cancer. Am J Transl Res 10: 866-874, 2018.

10. Deng L, Blanco FJ, Stevens H, Lu R, Caudrillier A, McBride M McClure JD, Grant J, Thomas M, Frid M, et al: MicroRNA-143 Activation regulates smooth muscle and endothelial cell crosstalk in pulmonary arterial hypertension. Circ Res 117: 870-883, 2015

11. Teague EM, Print CG and Hull ML: The role of microRNAs in endometriosis and associated reproductive conditions. Hum Reprod Update 16: 142-165, 2010.

12. Zheng B, Xue X, Zhao Y, Chen J, Xu CY and Duan P: The differential expression of microRNA-143,145 in endometriosis. Iran J Reprod Med 12: 555-560, 2014.

13. Cosar E, Mamillapalli R, Ersoy GS, Cho S, Seifer B and Taylor HS: Serum microRNAs as diagnostic markers of endometriosis: A comprehensive array-based analysis. Fertil Steril 106: 402-409, 2016.

14. Bravo-San Pedro JM, Kroemer G and Galluzzi L: Autophagy and mitophagy in cardiovascular disease. Circ Res 120: 1812-1824, 2017

15. Menzies FM, Fleming A, Caricasole A, Bento CF, Andrews SP, Ashkenazi A, Füllgrabe J, Jackson A, Jimenez Sanchez M, Karabiyik C, et al: Autophagy and neurodegeneration: Pathogenic mechanisms and therapeutic opportunities. Neuron 93: 1015-1034, 2017.

16. White E, Mehnert JM and Chan CS: Autophagy, metabolism, and cancer. Clin Cancer Res 21: 5037-5046, 2015.

17. Allavena G, Carrarelli P, Del Bello B, Luisi S, Petraglia F and Maellaro E: Autophagy is upregulated in ovarian endometriosis: A possible interplay with p53 and heme oxygenase-1. Fertil Steril 103: 1244-51.e1, 2015.

18. Neshkov NS: Use of ultrasound in therapy of neuroreceptor forms of impotence. Vopr Kurortol Fizioter Lech Fiz Kult 35 270, 1970 (In Russian).

19. Shi YL, Luo XZ, Zhu XY, Hua KQ, Zhu Y and Li DJ: Effects of combined 17beta-estradiol with TCDD on secretion of chemokine IL-8 and expression of its receptor CXCR1 in endometriotic focus-associated cells in co-culture. Hum Reprod 21 870-879, 2006

20. Livak KJ and Schmittgen TD: Analysis of relative gene expression data using real-time quantitative PCR and the 2(-Delta Delta C(T)) method. Methods 25: 402-408, 2001.

21. Mari-Alexandre J, Sanchez-Izquierdo D, Gilabert-Estelles J, Barcelo-Molina M, Braza-Boils A and Sandoval J: miRNAs regulation and its role as biomarkers in endometriosis. Int $\mathbf{J}$ Mol Sci 17: 93, 2016
22. Okamoto M, Nasu K, Abe W, Aoyagi Y, Kawano Y, Kai K, Moriyama M and Narahara H: Enhanced miR-210 expression promotes the pathogenesis of endometriosis through activation of signal transducer and activator of transcription 3 . Hum Reprod 30: 632-641, 2015.

23. Abdel-Rasheed, M., Nour Eldeen, G., Mahmoud, M., ElHefnawi, M., Abu-Shahba, N., Reda, M., Elsetohy, K., Nabil, M., Elnoury, et al: MicroRNA expression analysis in endometriotic serum treated mesenchymal stem cells. EXCLI journal 16: 852-867, 2017.

24. Ohlsson Teague EM, Van der Hoek KH, Van der Hoek MB, Perry N, Wagaarachchi P, Robertson SA, Print CG and Hull LM: MicroRNA-regulated pathways associated with endometriosis. Mol Endocrinol 23: 265-275, 2009.

25. He R, Liu X, Zhang J, Wang Z, Wang W, Fu L, Fan Y, Sun S, Cao Y, Zhan L, et al: NLRC5 inhibits inflammation of secretory phase ectopic endometrial stromal cells by up-regulating autophagy in ovarian endometriosis. Front Pharmacol 11: 1281, 2020.

26. Ding Y, Zhu Q, He Y, Lu Y, Wang Y, Qi J, Wu H, Xu R, Li J, $\mathrm{Li} \mathrm{X}$, et al: Induction of autophagy by Beclin-1 in granulosa cells contributes to follicular progesterone elevation in ovarian endometriosis. Transl Res 227: 15-29, 2021.

27. Ma W, Ding F, Wang X, Huang Q, Zhang L, Bi C, Hua B, Yuan Y, Han Z, Jin M, et al: By Targeting Atg7 MicroRNA-143 mediates oxidative stress-induced autophagy of $\mathrm{c}-\mathrm{Kit}^{+}$mouse cardiac progenitor cells. EBioMedicine 32: 182-191, 2018.

28. Lin XT, Zheng XB, Fan DJ, Yao QQ, Hu JC, Lian L, Wu XJ, Lan P and He XS: MicroRNA-143 targets ATG2B to inhibit autophagy and increase inflammatory responses in Crohn's disease. Inflamm Bowel Dis 24: 781-791, 2018

29. Wei J, Ma Z, Li Y, Zhao B, Wang D, Jin Y and Jin Y: miR-143 inhibits cell proliferation by targeting autophagy-related $2 \mathrm{~B}$ in non-small cell lung cancer H1299 cells. Mol Med Rep 11: 571-576, 2015.

30. Gao S, Wang K and Wang X: miR-375 targeting autophagyrelated 2B (ATG2B) suppresses autophagy and tumorigenesis in cisplatin-resistant osteosarcoma cells. Neoplasma 67: 724-734, 2020.

31. Tanbo T and Fedorcsak P: Endometriosis-associated infertility: Aspects of pathophysiological mechanisms and treatment options. Acta Obstet Gynecol Scand 96: 659-667, 2017.

32. Ahn SH, Monsanto SP, Miller C, Singh SS, Thomas R and Tayade C: Pathophysiology and immune dysfunction in endometriosis. BioMed Res Int 2015: 795976, 2015

33. Králíčková $M$ and Vetvicka V: Immunological aspects of endometriosis: A review. Ann Transl Med 3: 153, 2015.

34. Olovsson M: Immunological aspects of endometriosis: An update. Am J Reprod Immunol 66 (Suppl 1): 101-104, 2011.

35. Filippi I, Carrarelli P, Luisi S, Batteux F, Chapron C, Naldini A and Petraglia F: Different expression of hypoxic and angiogenic factors in human endometriotic lesions. Reprod Sci 23: 492-497, 2016.

36. Zhan L, Wang W, Zhang Y, Song E, Fan Y and Wei B: Hypoxia-inducible factor-1alpha: A promising therapeutic target in endometriosis. Biochimie 123: 130-137, 2016.

37. Tsuzuki T, Okada H, Cho H, Tsuji S, Nishigaki A, Yasuda K and Kanzaki H: Hypoxic stress simultaneously stimulates vascular endothelial growth factor via hypoxia-inducible factor-1 $\alpha$ and inhibits stromal cell-derived factor-1 in human endometrial stromal cells. Hum Reprod 27: 523-530, 2012

38. Daskalaki I, Gkikas I and Tavernarakis N: Hypoxia and selective autophagy in cancer development and therapy. Front Cell Dev Biol 6: 104, 2018

39. Liu W, Meng Y, Zong C, Zhang S and Wei L: Autophagy and tumorigenesis. Adv Exp Med Biol 1207: 275-299, 2020.

40. White E: The role for autophagy in cancer. J Clin Invest 125: 42-46, 2015.

41. Leyendecker G, Kunz G, Noe M, Herbertz M and Mall G: Endometriosis: A dysfunction and disease of the archimetra. Hum Reprod Update 4: 752-762, 1998.

42. Ruiz A, Rockfield S, Taran N, Haller E, Engelman RW, Flores I, Panina-Bordignon P and Nanjundan M: Effect of hydroxychloroquine and characterization of autophagy in a mouse model of endometriosis. Cell Death Dis 7: e2059, 2016.

43. Zhan L, Li J and Wei B: Autophagy in endometriosis: Friend or foe? Biochem Biophys Res Commun 495: 60-63, 2018.

44. Liu H, Zhang Z, Xiong W, Zhang L, Xiong Y, Li N, He H, Du Y, Liu Y. Hypoxia-inducible factor-1alpha promotes endometrial stromal cells migration and invasion by upregulating autophagy in endometriosis. Reproduction 153: 809-820, 2017. 
45. Pei T, Huang X, Long Y, Duan C, Liu T, Li Y and Huang W: Increased expression of YAP is associated with decreased cell autophagy in the eutopic endometrial stromal cells of endometriosis. Mol Cell Endocrinol 491: 110432, 2019.

46. Sui X, Li Y, Sun Y, Li C, Li X and Zhang G: Expression and significance of autophagy genes LC3, Beclin1 and MMP-2 in endometriosis. Exp Ther Med 16: 1958-1962, 2018.

47. Mei J, Zhou WJ, Zhu XY, Lu H, Wu K, Yang HL, Fu Q, Wei CY Chang KK, Jin LP, et al: Suppression of autophagy and HCK signaling promotes PTGS2high FCGR3- NK cell differentiation triggered by ectopic endometrial stromal cells. Autophagy 14: $1376-1397,2018$.

48. Luo X, Cheng W, Wang S, Chen Z and Tan J: Autophagy suppresses invasiveness of endometrial cells through reduction of Fascin-1. BioMed Res Int 2018: 8615435, 2018.

49. Zhao Y and Srivastava D: A developmental view of microRNA function. Trends Biochem Sci 32: 189-197, 2007.

50. Esquela-Kerscher A and Slack FJ: Oncomirs - microRNAs with a role in cancer. Nat Rev Cancer 6: 259-269, 2006.

51. Stolzenburg LR and Harris A: The role of microRNAs in chronic respiratory disease: Recent insights. Biol Chem 399: 219-234, 2018.
52. Soroosh A, Koutsioumpa M, Pothoulakis C and Iliopoulos D: Functional role and therapeutic targeting of microRNAs in inflammatory bowel disease. Am J Physiol Gastrointest Liver Physiol 314: G256-G262, 2018.

53. Wu MH, Chen KF, Lin SC, Lgu CW and Tsai SJ: Aberrant expression of leptin in human endometriotic stromal cells is induced by elevated levels of hypoxia inducible factor-1alpha. Am J Pathol 170: 590-598, 2007.

54. Hsiao KY, Lin SC, Wu MH and Tsai SJ: Pathological functions of hypoxia in endometriosis. Front Biosci (Elite Ed) 7: 309-321, 2015.

55. Donnez J, Smoes P, Gillerot S, Casanas-Roux F and Nisolle M: Vascular endothelial growth factor (VEGF) in endometriosis. Hum Reprod 13: 1686-1690, 1998.

56. Zhang X, Liu S, Hu T, Liu S, He Y and Sun S: Up-regulated microRNA-143 transcribed by nuclear factor kappa B enhances hepatocarcinoma metastasis by repressing fibronectin expression. Hepatology 50: 490-499, 2009.

(i) (1) $($ This work is licensed under a Creative Commons

EY NG ND Attribution-NonCommercial-NoDerivatives 4.0 International (CC BY-NC-ND 4.0) License. 